\title{
Brief counselling by a primary care physician or nurse practitioner reduced alcohol consumption in high risk drinkers
}

Ockene JK, Adams A, Hurley T, et al. Brief physician- and nurse practitioner-delivered counseling for high-risk drinkers. Does it work? Arch Intern Med 1999;159:2198-205.

QUESTION: Is brief counselling given by a physician or nurse practitioner during a routine outpatient visit effective in reducing alcohol consumption among high risk drinkers?

\section{Design}

Randomised (unclear allocation concealment), blinded (outcome assessors), controlled trial with 6 months of follow up.

\section{Setting}

4 primary care internal medicine sites at a university medical centre in Massachusetts, USA.

\section{Patients}

530 patients between 21 and 70 years of age (mean age $44 \mathrm{y}, 65 \%$ men) who were scheduled to be seen at 1 of the 4 primary care sites and who were high risk drinkers (men who drank $>12$ drinks/wk or binged on $\geqslant 5$ drinks on $\geqslant 1$ occasion in the previous month; or women who drank $>9$ drinks/wk or binged on $\geqslant 4$ drinks on $\geqslant 1$ occasion in the previous month). Patients were excluded if they were pregnant, planned to move out of the area within 1 year, did not have a telephone, were already in an alcohol intervention programme, or had an Axis I psychiatric disorder. Follow up was $91 \%$.

\section{Intervention}

The 4 primary care sites (38 physicians and 8 nurse practitioners) were randomised to the intervention $(\mathrm{n}=274$ patients $)$ or usual care $(\mathrm{n}=256$ patients). Physicians and nurse practitioners in the intervention group had 2.5 hours of training on the patient centred alcohol intervention programme. Patients in the intervention group received a health booklet with advice on general health issues and a 5-10 minute counselling session at the time of their routine visit. Patients in the usual care group received the same health booklet and were encouraged to direct any health questions they had to their physician or nurse practitioner. All physicians and nurse practitioners were encouraged to attend weekly conferences that included biannual presentations on managing patients with alcohol problems.

\section{Main outcome measure}

Change in alcohol use (weekly alcohol use and Source of funding: National Institute on Alcohol Abuse and Alcoholism.

For correspondence: DrJ K Ockene, Division of Preventive and Behavioral Medicine University of

Massachusetts Medical School, 55 Lake Ave N, Worcester, MA 01655. Fax +15088561570 . frequency of binge drinking episodes/mo) at 6 months.

\section{Main results}

After adjusting for age, sex, and baseline alcohol consumption, patients who received the intervention had lower alcohol consumption than those who received usual care (mean reduction $5.8 v 3.4$ drinks/wk, $p=0.001)$. No difference existed for mean reduction in binge drinking episodes per month between patients in the intervention group and those in the usual care group (1.8 $v 1.0, \mathrm{p}=0.09)$.

\section{Conclusion}

Brief counselling given by a physician or nurse practitioner as part of routine primary care reduced alcohol consumption by high risk drinkers.

\section{COMMENTARY}

Ockene $e t$ al add to the growing body of work about brief interventions that are effective in reducing alcohol consumption among high risk drinkers. ${ }^{1}$ The authors note that high risk drinking is a serious public health problem resulting in alcohol related deaths and hospital costs because of alcohol related illnesses. The development of an economical and effective intervention that can be delivered at a primary healthcare level to manage alcohol use by high risk drinkers is important.

The brief counselling intervention used by the physicians or nurse practitioners was patient centred and aimed to empower patients to meet their treatment goals. At 6 months, there was almost a 2 fold adjusted mean reduction in weekly alcohol use for those who received the intervention. This effect is particularly impressive given that $41 \%$ of the participants received only one 5-10 minute counselling session.

Study strengths included the avoidance of contamination by randomising practices rather than patients, accounting for the unit of randomisation in the analysis, and a high follow up rate $(91 \%)$ of study participants. The study also had several limitations. The baseline level for high risk drinking appears low and participants who were identified to be high risk drinkers may not have viewed themselves as such. The study excluded people without a telephone, those who were already in an alcohol programme, and those who had a psychiatric disorder. The sample included few people from minority groups. These sampling issues may limit the generalisability of the study findings. The change in alcohol use was measured at 6 months and it would be interesting to see if the brief counselling session continued to be effective at a longer follow up point.

A previous study showed that it was possible to identify people who were high risk drinkers within the primary care setting through the use of a relatively simple screening tool. ${ }^{2}$ The study by Ockene et al showed that it was possible, through the use of a patient centred approach, to provide high risk drinkers with motivation to meet their alcohol treatment goals.

Robert Johnson, RGN, RMN Community Psychiatric Nurse for Alcohol and Drugs Highland Primary Care NHS Trust

Wick, UK

1 Bien TH, Miller WR, Tonigan JS. Brief interventions for alcohol problems: a review. Addiction 1993;88:315-35.

2 Saunders JB, Aasland OG, Amundsen A, et al. Alcohol consumption and related problems among primary health care patients: WHO collaborative project on early detection of persons with harmful alcohol consumption-I. Addiction 1993;88:349-62. 